\title{
Graphene Nanoplatelets (GnP)-PVA Based Passive Saturable Absorber
}

\author{
Nabihah Hussin ${ }^{1}$, Mohd Haniff Ibrahim ${ }^{2}$, Fauzan Ahmad ${ }^{\star 3}$, Hafizal Yahaya ${ }^{4}$, \\ Sulaiman Wadi Harun ${ }^{5}$ \\ ${ }^{1,2}$ Faculty of Electrical Engineering, Universiti Teknologi Malaysia, 81310 Skudai, Johor, malaysia \\ 3.4 Malaysia Japan International Institute of Technology (MJIIT), Universiti Teknologi Malaysia, 54100 \\ Kuala Lumpur, Malaysia \\ ${ }^{5}$ Photonic Research Center, University of Malaya, 50603 Kuala Lumpur, Malaysia \\ ${ }^{*}$ Corresponding author, e-mail: fauzan.kl@utm.my
}

\begin{abstract}
A passive Q-switched pulsed laser at $1.5 \mu \mathrm{m}$ region incorporating graphene nanoplatelets (GnPs) embedded in Polyvinyl Alcohol (PVA) is demonstrated. A surfactant is used to aid the dispersion of the GnPs before it is mixed with PVA to develop a GnPs-PVA film based SA. The SA is integrated into the laser cavity by attaching a cut of the GnPs-PVA film in between two fiber ferrule of the laser ring cavity. The proposed GnPs-PVA film based passive Q-switched laser was able to operate as the input pump power was increased from $39 \mathrm{~mW}$ up to a maximum of $148 \mathrm{~mW}$ before diminishing. The laser obtained operated with a central wavelength of $1530.76 \mathrm{~nm}$. Repetition rates were obtained at $33 \mathrm{kHz}$ to $91.5 \mathrm{kHz}$, throughout the tunable input pump power with the shortest pulse width of $2.42 \mu \mathrm{s}$. Maximum attainable peak power and pulse energy of $1.2 \mathrm{~mW}$ and $5.9 \mathrm{~nJ}$, respectively, was recorded, accompanied by a signal to noise ratio (SNR) of $28 \mathrm{~dB}$.
\end{abstract}

Keywords: graphene nanoplatelets, saturable absorber, Q-switched laser

Copyright $\odot 2017$ Universitas Ahmad Dahlan. All rights reserved.

\section{Introduction}

Erbium-doped fiber lasers (EDFLs) which works in Q-switched regime provide a light sources with potential alication in optical communication, laser range finding and material processing [1-3]. There are two common aroach to generate pulse fiber laser, active and passive Q-switching, with the latter provide several advantages such as low-cost, compact and simple circuitry. Basically, the passive saturable absorber (SA) is to reside light dependent intensity material in the laser cavity which enables the high-intensity components of an optical pulse to pass through, while the lower intensity components was blocked depends on the relaxation time of the materials. The material's level of ability when acting as a saturable absorber is highly dependable on their high nonlinearities, ultrafast carrier relaxation and broadband operating wavelength [4,5]. Different types of SA has been proposed and demonstrated for Q-switched fiber laser including carbon nanotubes (CNT), semiconductor saturable absorber mirrors (SESAM) and 2D based materials such as Topological insulators (Tis), graphene, and transition metal disulphides [6-8]. The distinct advantage of graphene based SA over other materials is its extremely broad operation bandwidth.

Graphite is when multiples of hexagon sheets stacked onto each other. Removing one of the hexagon sheets will results in a layer of graphite sheet called graphene [9-10]. The one atom thick material was proven to absorb a high fraction of incident white light [11] which is highly attributed to its unique electronic properties. Since graphene can be stacked to form graphites, the optical properties of it also changes with the changing layers [12-13] which makes it even more versatile and unique. Currently, graphene based SA for Q-switched pulse laser was demonstrated using CVD approach, graphene nanocomposites, graphene oxide and reduce graphene oxide [14-19].

The graphene chemical vapor deposition (CVD) approach for Q-switched pulse laser has been demonstrated in [14] by placing a graphene based thin film which is produced via CVD between two fiber connectors. They reported to produce repetition rate between $34.72 \mathrm{kHz}$ to $53.2 \mathrm{kHz}$ with the shortest pulse with of $3.2 \mu \mathrm{s}$, within input pump power from $57.2 \mathrm{~mW}$ to 
$74.23 \mathrm{~mW}$. Popa et al [15] demonstrated liquid phase exfoliation route to fabricate graphenePVA nanocomposites based passive saturable absorber with tunable filter to tune the central wavelength. The tuneable central wavelength is from 1522nm to $1555 \mathrm{~nm}$ with threshold of pump power of $74 \mathrm{~mW}$. Meanwhile, Ahmad et al [16] fabricate a graphene based SA by optical deposition of graphene- N-methyl Pyrrolidone (NMP) suspension injected with $11 \mathrm{dBm}$ of 1550 $\mathrm{nm}$ laser source. They demonstrated tunable wavelength from $1547.66 \mathrm{~nm}$ to $1557.66 \mathrm{~nm}$ using fiber Bragg grating as tuning element's with threshold input pump power of $39.8 \mathrm{~mW}$. A wider tunable range of $50 \mathrm{~nm}$ was reported in [17] by optical deposition of grapheneDimethylformamide (DMF) suspension. The SA works within input power from $33 \mathrm{~mW}$ to $61 \mathrm{~mW}$ and produced maximum repetition rate of $29.05 \mathrm{kHz}$ with shortest pulse width of $4.6 \mu \mathrm{s}$. Yap et al, [18] reported a simple aroach to fabricate Graphene oxide (GO) based SA by diing a fiber ferrule end face onto the GO suspension and integrated in Erbium doped fiber laser in ring cavity. The reported threshold input pump power for pulse laser generation is $9.5 \mathrm{~mW}$ with range of repetition and shortest pulse width of 16.0 to $57.0 \mathrm{kHz}$ and $3.90 \mu \mathrm{s}$, respectively. A higher threshold of $120 \mathrm{~mW}$ was reported for reduced graphene oxide (rGO) based SA as reported in [19], in the expense of $1.85 \mu$ s temporal pulse width and $125 \mathrm{~nJ}$ of pulse energy.

Graphene nanoplatelets (GnPs) is the derivative of graphene, with 3-10 stacks of graphene [20], while graphene with number of layer higher than 10 is define as graphite. In comparison to Graphene Oxide (GO), the quality of GnPs is same as graphene due to the similarity configuration of pristine basal plane with improve optical, electrical, and mechanical properties [20]. GnPs has been used as filler to improve the thermal and mechanical properties of composites material [21] and low cost fuel cell base material [22], but the alication on optical properties is not intensively reported compared to graphene and graphene oxide. One of the interesting points is that the GnPs film consists of multiple layer of graphene and rather than control the layer numbers by overlaing two to three films produced by CVD as demonstrated by Sun [23].

\section{Research Method}

Graphene nanoplatelets (GnPs) powder was obtained from Low Dimensional Material Research Center (LDMRC) University of Malaya. The dispersion of GnPs was achieved by adding $40 \mathrm{mg}$ of $\mathrm{GnP}$ nano powder in $40 \mathrm{ml}$ of $1 \%$ of sodium dodecyl sulfate (SDS) in deionized (DI) water, and then sonicated for one hour at $50 \mathrm{~W}$. The large particles of GnPs in the suspension are taken away by centrifugation at $1000 \mathrm{rpm}$. The resulted dispersed suspension was added into a PVA solution in one-to-one ratio. The PVA solution was prepared separately at first, by dissolving $1 \mathrm{~g}$ of polyvinyl alcohol (PVA) ( $\left.\mathrm{Mw}=89 \times 10^{3} \mathrm{~g} / \mathrm{Mol}\right)$ (Sigma Aldrich) in 120 $\mathrm{ml}$ of DI water. The homogeneous GnPs-PVA composites were obtained by a one hour ultrasonic process. After that, the GnPs-PVA suspension were poured on a petri dish where it is left at room temperature for 48 hours to develop a GnPs-PVA film with a measured thickness of around $50 \mu \mathrm{m}$. The morphology of the developed film then was investigated using FieldEmission Scanning Electron Microscope (FESEM) (Hitachi SU8020) as shown in Figure 1. The captured image showed a thoroughly mixed of GnPs in PVA polymer with smooth surface and low aggregation. A small portion of the developed GnPs-PVA film was attached to the end of fiber ferrule and then integrated in the laser cavity assisted by fiber connector. The experimental setup of the pulsed laser with the Erbium doped fiber (EDF) as gain medium is illustrated as seen in Figure 2. The core and cladding diameter of the EDF is $8 \mu \mathrm{m}$ and $125 \mu \mathrm{m}$, respectively, with a numerical aperture (NA) of 0.16 . At $980 \mathrm{~nm}$ and $1530 \mathrm{~nm}$, the rate of ion absoprtions is at $45 \mathrm{~dB} / \mathrm{m}$ and $80 \mathrm{~dB} . \mathrm{m}$, respectively. As shown in the figure, the laser cavity is pumped by a 980 $\mathrm{nm}$ laser diode via a 980/1550 nm wavelength division multiplexer (WDM), an Erbium doped fiber with the length of $1 \mathrm{~m}$, an isolator to prevent any backflow of the light oscillation during laser generation, the newly fabricated GnPs-PVA based thin film, and a 95/5 output coupler. The output coupler is needed to tap out $5 \%$ of the output for characterization purposes through a $3 \mathrm{~dB}$ coupler. The components were arranged in a ring configuration to allow for continuous light oscillations. An optical spectrum analyser (OSA) is used to observe the laser's spectrum with $0.05 \mathrm{~nm}$ spectral resolution while an oscilloscope is used to measure the laser's repetition rates and pulse widths. The optical power meter (OPM) and the radio frequency spectrum analyser (RFSA) is used to measure the output power and signal-to-noise ratio (SNR) of the output laser, respectively. 


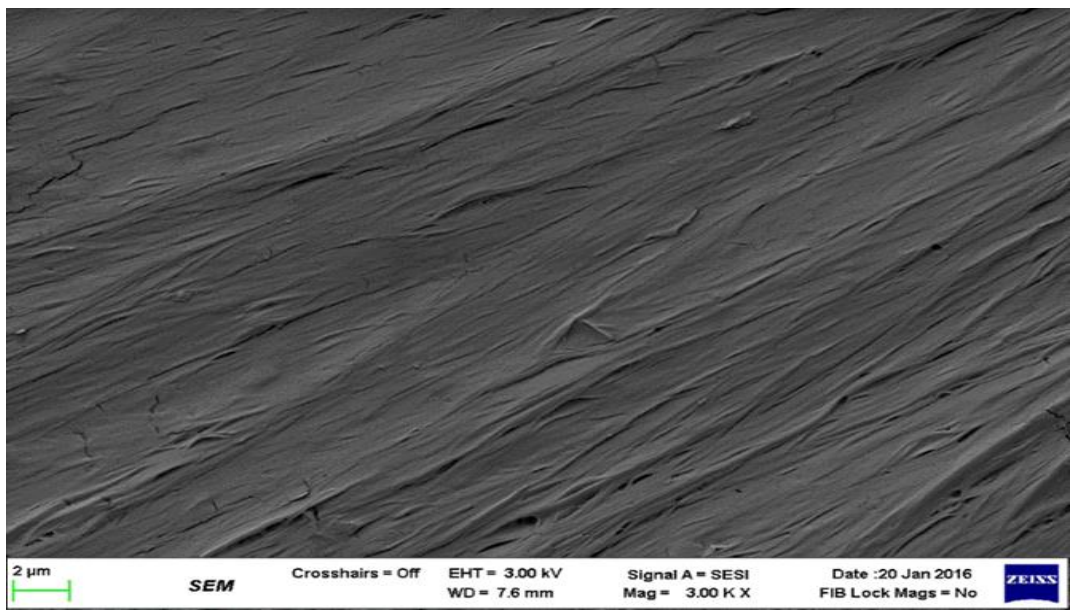

Figure 1. Graphene Nanoplatelets (GnP)-PVA

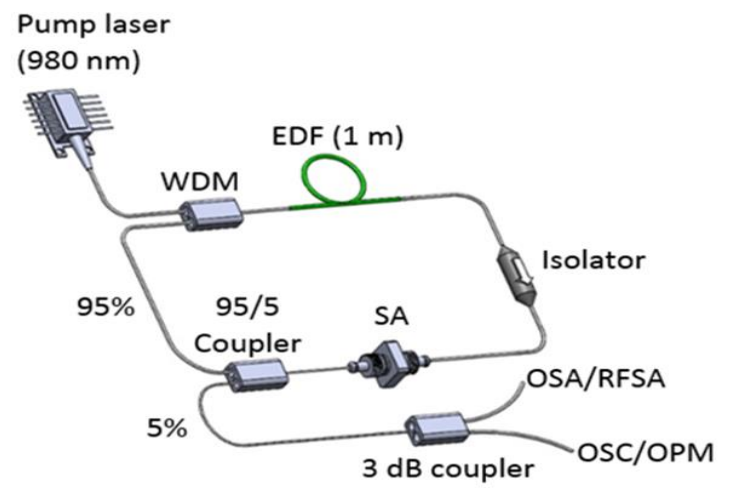

Figure 2. Integration of GnP-PVA film based SA in fiber laser in ring cavity

\section{Results and Analysis}

The experimental results revealed the central operating wavelength for continuous wave lasing is at $1559.19 \mathrm{~nm}$. The integration of GnPs-PVA film based saturable absorber (SA) in the laser cavity produced Q-switched pulsed laser with threshold input pump power of 39 $\mathrm{mW}$. The generated Q-switched pulse laser shifted the central wavelength to $1530.76 \mathrm{~nm}$ with 3-dB spectral broadening around $1 \mathrm{~nm}$ due to loss induced by the passive saturable absorber as shown in Figure 3. The eution of the time domain within input pump range from $39 \mathrm{~mW}$ to $148 \mathrm{~mW}$. Beyond $148 \mathrm{~mW}$, there is no pulse generated due to the GnP-PVA SA is fully saturated or dislocated due to induced heat and pressure at high input pump power. There is no pulse laser observed when the GnPs-PVA film based SA was removed from laser cavity, confirming the sole effect of GnP-PVA SA for pulse laser generation. Furthermore, the pulse train of the generated pulse was recorded using the oscilloscope for the range of input pump power from $39 \mathrm{~mW}$ to $148 \mathrm{~mW}$. Figure 4 (a) and (b) shows the recorded pulse train at threshold and the maximum input pump power, with respective pulse width. The generated pulse train shows a stable pulse train with no peak fluctuation at threshold input pump power and at maximum input pump power. 


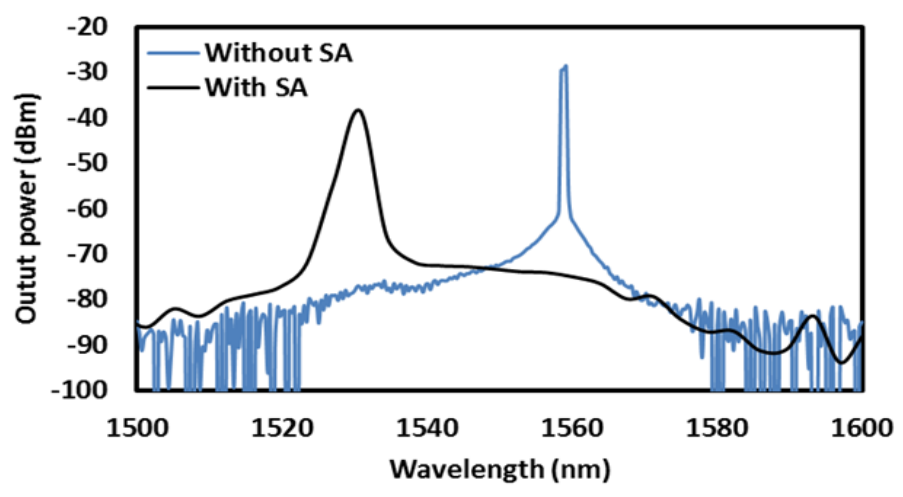

Figure 3. OSA trace without SA and with SA
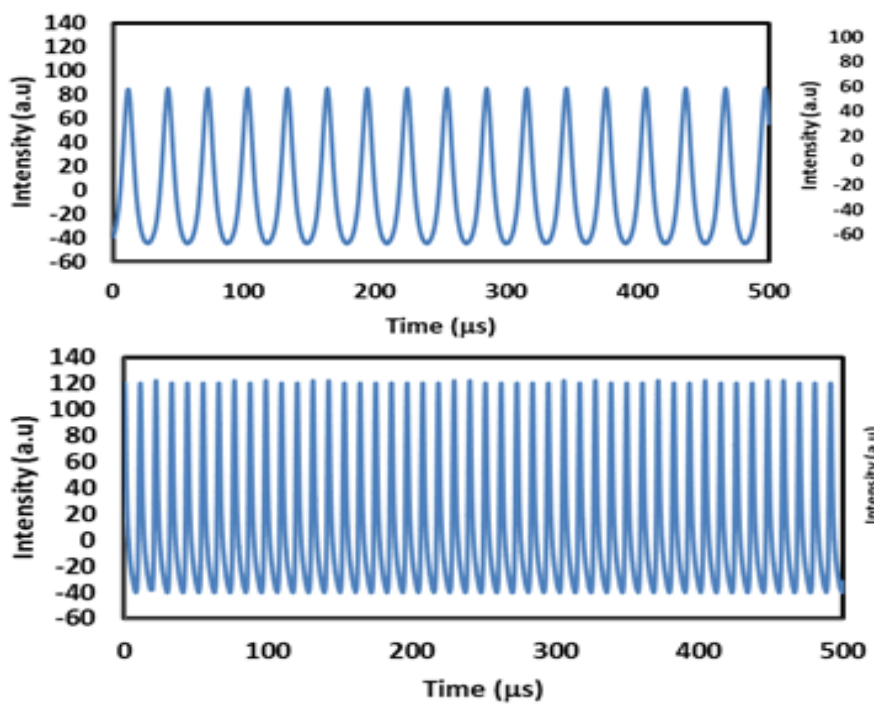
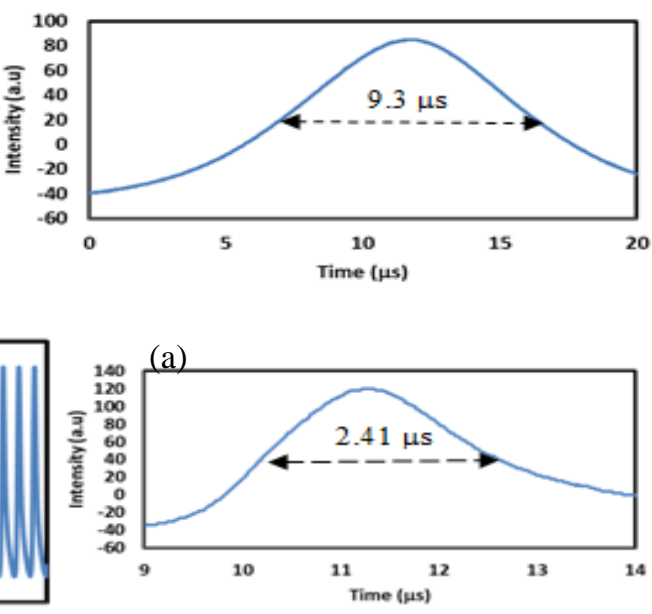

Figure 4. (a) At threshold pump power of $39.1 \mathrm{~mW}$ with pulse tfain of $32.2 \mathrm{kHz}$ and single pulse envelope with pulse width of $9.3 \mu \mathrm{s}$ (b) At maximum pump power of $148.7 \mathrm{~mW}$ with pulse train of $91 \mathrm{kHz}$ and single pulse envelope with pulse width of $2.41 \mu \mathrm{s}$

Figure 5 shows the measured generated pulse train with the respective pulse width in the function of input pump power. The repetition rate increase from $33 \mathrm{kHz}$ to $91.5 \mathrm{kHz}$ when the input pump power tuned from $39 \mathrm{~mW}$ to $148 \mathrm{~mW}$. The pulse width shows the decreasing trend from $9.3 \mu \mathrm{s}$ to $2.41 \mu \mathrm{s}$ with the increasing input pump power. This observation corresponds to the decreasing pulse width with the increasing repetition rates which commonly observed in passively $Q$-switched lasers [24]. The output average power $\left(P_{\text {av }}\right)$ was measured with an optical power meter. The pulse width $\left(\Delta \mathrm{t}_{\mathrm{p}}\right)$ and the pulse repetition rate $(f)$ were observed using digital oscilloscope via photo-detector. From the recorded data, pulse energy and instantaneous peak power is calculated using formula of $\mathrm{E}=\mathrm{P}_{\mathrm{av}} / f$ and eak $=\mathrm{E} / \Delta \mathrm{t}_{\mathrm{p}}$. Figure 6 shows the calculated peak power and pulse energy as function of pump power. The calculated peak power and pulse energy shows an increasing trend for input pump power varied from 39 $\mathrm{mW}$ to $104 \mathrm{~mW}$. The maximum peak power and pulse energy is $1.2 \mathrm{~mW}$ and $5.9 \mathrm{~nJ}$, respectively at input pump power of $104 \mathrm{~mW}$. Beyond $104 \mathrm{~mW}$ input pump power, the peak power and pulse energy is toward decreasing trends due to decreasing of the recorded average output power. Figure 7 shows the stability of the generated pulse using Radio frequency spectrum analyzer (RFSA) with the span of $500 \mathrm{kHz}$. The first beat note is around $28 \mathrm{~dB}$ and the generated pulse laser propagates with high stability as shown with the stable peak of the recorded RFSA. 


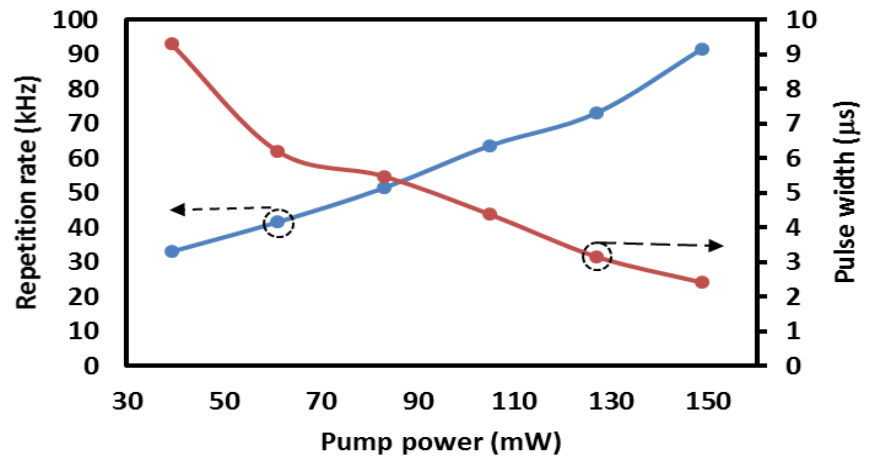

Figure 5. Repetition rate and pulse width as function of pump power

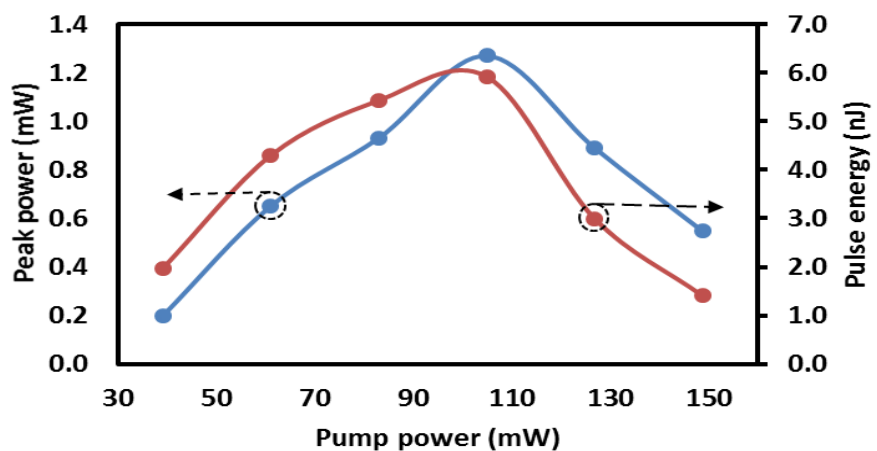

Figure 6. Peak power and pulse energy as function of pump power

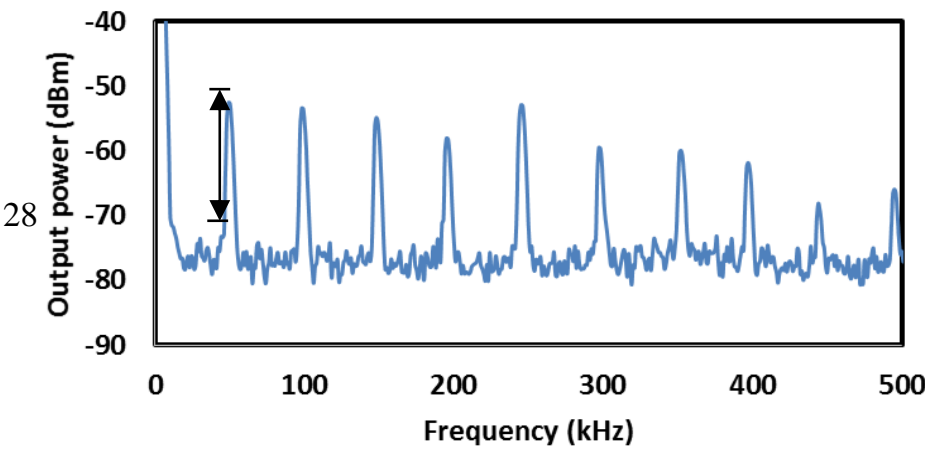

Figure 7. RFSA measurement for repetition rate of $50 \mathrm{kHz}$ with $500 \mathrm{kHz}$ span, the first beat note of $28 \mathrm{~dB}$

\section{Conclusion}

Graphene nanoplatelets-PVA (GnP-PVA) film based passive Q-switcher was successfully demonstrated at $1.5 \mu \mathrm{m}$ region. The solution casting aroach at room temperature in fabricating GnPs-PVA based saturable absorber is simple, low cost and offers a scalable production of the SA. Experimental works shows the capability of the developed GnPs-PVA film to act as passive Q-switcher when resides in Erbium doped fiber laser (EDFL). The film able to operates to the maximum input pump power of $148 \mathrm{~mW}$, maximum repetition rates of $91.5 \mathrm{kHz}$ and shortest pulse width of $2.42 \mu \mathrm{s}$. The calculated output peak power and pulse energy of 1.2 $\mathrm{mW}$ and $5.9 \mathrm{~nJ}$, can be use in meteorology and material processing. 


\section{References}

[1] ME Fermann, I Hartl. Ultrafast fibre lasers, Nature Photon. 2013; 7(11): 868-874.

[2] FW Wise, A Chong, WH Renninger. High-energy femtosecond iber lasers based on pulse propagation at normal dispersion. Laser Photon. Rev. 2008; 2(1/2): 58-73.

[3] SD Jackson Towards high-power mid-infrared emission from a fibre laser, Nature Photon. 2012; 6(7): 423-43.

[4] T Hasan, Z Sun, F Wang, F Bonacrosso, PH Tan, AG Rozhin, AC Ferrari. Nanotube-Polymer Composites for Ultrafast Photonics. Adv. Mat. 2009; 21: 3874-3899.

[5] F Bonaccorso, Z Sun, T Hasan, AC Ferrari. Graphene photonics and optoelectronics. Nat. Photon. 2010; 4(9); 611-622.

[6] Z Sun, T Hasan, AC Ferrari. Ultrafast lasers mode-locked by nanotubes and grapheme. Physica E. 2012; 44: 1082-1091.

[7] U Keller et al. Semiconductor Saturable Absorber Mirrors (SESAM's) for femtosecond to nanosecond pulse generation in solid-state lasers. IEEE J. Sel. Topics Quantum Electron. 1996; 2(3): 435-453.

[8] G Sobon. Mode-locking of fiber lasers using novel two-dimensional nanomaterials: graphene and topological insulators. Photon. Res. 2015; 3(2): A56-A63.

[9] MJ Allen, VC Tung, RB Kaner, Honeycomb Carbon: A Review of Graphene.Chemical Reviews. 2010; 110(1): 132-145.

[10] AK Geim. Graphene: Status and Prospects, Science. 2009; 324(5934): 1530-1534.

[11] RR Nair, P Blake, AN Grigorenko, KS Novoselov, TJ Booth, T Stauber, NMR Peres, AK Geim. Fine Structure Constant Defines Visual Transparency of Graphene. Science. 2008; 320(5881): 1308.

[12] AB Kuzmenko, E van Heumen, F Carbone, D van der Marel. Universal Optical Conductance of Graphite. Physical Review Letters. 2008; 100(11): 117401.

[13] S Yamashita. A Tutorial on Nonlinear Photonic Alications of Carbon Nanotube and Graphene, Journal of Lightwave Technology. 2012; 30(4): 427-447.

[14] LQ Zhang, Z Zhuo, JX Wang, YZ. Wang Passively Q-switched Fiber Laser Based on Graphene Saturable Absorber. Laser Physics. 2012; 22(2): 433-436.

[15] D Popa, Z Sun, T Hasan, F Torrisi, F Wang, AC Ferrari. Graphene Q-switched, tunable fiber laser, Alied Physics Letters. 2011; 98(7): 073106- 073106.

[16] H Ahmad, MZ Zulkifli, FD Muhammad, AZ Zulkifli, SW Harun. Tunable graphene-based Q-switched erbium-doped fiber laser using fiber Bragg grating. Journal of Modern Optics. 2013; 60(3): 202-212.

[17] WJ Cao, HY Wang, AP Luo, ZC Luo, WC Xu, Graphene based, $50 \mathrm{~nm}$ wide-band tunable passively Q-switched fiber laser. Laser Physics Letters. 2012; 9(1): 54-58.

[18] YK Yap, N M Huang, SW Harun, H Ahmad, Graphene Oxide-Based Q-Switched Erbium-Doped Fiber Laser Chin. Phys. Lett. 2013; 30(2): 024208.

[19] Gzegorz Sobon et al. Linearly polarized, Q-switched Er-doped fiber laser based on reduced graphene oxide saturable absorber. Al. Phys. Lett. 2012; 101; 241106.

[20] SN Kazi et al. Investigation on the use of graphene oxide as novel surfactant to stabilize weakly charged graphene nanoplatelets. Nanoscale Research Letters. 2015; 10: 212.

[21] J Jeon, SG Jeong, JH Lee, J Seo, S Kim. High thermal performance composite PCMs loading xGnP for alication to building using radiant floor heating system. Solar Energy Mater Solar Cells. 2012; 101: 51-60.

[22] IH Do. Metal Decoration of exfoliated graphite nanoplatelets (xGnP) for fuel cell alications, Ph.D. Dissertation; Michigan State University, East Lansing, Michigan. 2006.

[23] B Fu et al. Broadband Graphene Saturable Absorber for Pulsed Fiber Lasers at 1, 1.5, and $2 \mu \mathrm{m}$, IEEE Journal of Selected Topics in Quantum Electronics. 2014; 20(5)

[24] XC Lin et al. Multi-walled carbon nanotube as a saturable absorber for a passively mode-locked $\mathrm{Nd}: \mathrm{YVO}_{4}$ laser. Laser Phys. Lett. 2013; 10: 055805(4). 